\title{
PERFORMANCE STUDIES ON WATER FLOW CONTROL USING $P$, PI AND PID CONTROLLERS
}

\author{
Ramesh Babu Aremanda*, ORCID ID: 0000-0002-6609-8640, \\ Nahom Yohannes, ORCID ID: 0000-0001-8926-5141, \\ Yosief Ghezae, ORCID ID: 0000-0001-9602-3546

\begin{abstract}
Department of Chemical Engineering, Mai Nefhi College of Engineering \& Technology (MCOETEC), P.O. Box 344, Asmara, Eritrea.

${ }^{*}$ Corresponding author: Ramesh Babu Aremanda, ramesh.nitrkl@gmail.com
\end{abstract}

Received: 05. 28. 2021

Accepted: 08.11. 2021

\begin{abstract}
The main purpose of this study is to revitalize the concept of wise and controlled supply of water for domestic, industrial and agricultural applications which facilitate sustainable usage of fresh water resources. As Eritrea is striving to manage its water resources, attention paid primarily to enable water flow control mechanisms in municipal water distribution systems. A table top process control trainer (PCT) was tested through proportional(P), integral (I) and derivative (D) control mechanisms using Ziegler-Nichols second method to evaluate the tuning variables. Applying exclusively $\mathrm{P}$ control action, critical period of oscillation $\left(P_{c r}\right)$ was estimated as $1.4 \mathrm{sec}$ at proportional band value of 9 . $\mathrm{P}, \mathrm{PI}$ and PID controller performance studies were conducted with tuned variables on the water flow control system at different step disturbances between $20-50 \%$ and their corresponding responses were characterized. $\mathrm{P}$ controller exhibited faster responses with consistent increments in offset, PI controller recorded highest overshoot values with negligible offset and prolonged settling times. PID controller showed less overshoot values and faster response times than PI but it increased chatter on the control output signal. The study revealed that the system can be safely controlled between 0-80 LPH. If the offset is not a major concern, $\mathrm{P}$ controller would be reflected suitable with simple design and minimum expenditure, else PI controller makes offset to zero though it possesses higher settling times. In other words, PID controller is complex using more tuning parameters, need expensive maintenance, and has resulted an intermittent noise in the output signal.
\end{abstract}

Keywords: Control System, Flow controllers, Step Disturbances, Sustainable Supply of Water, Tuning of PID Controllers, Water Flow control, Ziegler and Nichols Method.

\section{Introduction}

Freshwater supply is the main pillar for sustainable economic activities of humankind and an essential element of human life. Currently, scarcity and quality of water have become prior concerns in many developing countries. Of the $3 \%$ of freshwater on earth, only one third is of drinking water quality available in streams, rivers and lakes that can support human's 
daily activities and other usage [1]. Globally, water use has been increasing by $1 \%$ every year and expected to rise 20 - $30 \%$ by 2050 [2]. Population growth and urbanization are prominent reasons for water scarcity everywhere in the world [3, 4]. Eritrea, a northeast African country mainly relies on seasonal rainfalls usually happens every year for short period from July to September. However, Government of Eritrea (GOE) has made significant progress in water and sanitation service delivery since its independence. Despite progress, water scarcity and poor water quality are increasingly common across Eritrea, demand for freshwater in rural and urban areas is expected to increase as a result of rapid climate change, population growth, rapid urbanization, economic activity, competition for water and improved standards of living. Hence, in 2019, with the support of UNICEF, the GOE has developed a One WASH (Water, Sanitation and Hygiene) Strategy and Investment Plan (OWSIP) that integrates resilience considerations, climate change mitigation and adaptation [5]. Therefore, the study of water supply control systems and their design strategies would be on prior to ensure deterministic supply with improved safety.

Flow controllers are designed to measure and control the flow of liquids and gases in domestic, industrial and agricultural applications. Water flow control in process industries is essential for precise supply in manufacturing operations, perhaps it may decide the end product specification. An effective fluid control equipment can also help in the prevention of hazardous substances into the working environment. Flow controllers, which need modulating electronically to enable a closed process loop are installed to speed up and improve the processes whilst lowering the cost. Applications that benefit from this include the sampling systems in petrochemical plants, gas and chemical control in manufacturing processes and in off-shore oil rigs. Solenoid valves are a good performer in these environments; however, it is important to consider the power requirements and the environment they are being used in. A standard solenoid would potentially pose great risk without being upgraded, due to the electricity needed to operate them. A simple and more reliable method of flow control within these environments are pneumatic valves. The removal of electricity from its process of delivery, removes fire and explosion hazards [6, 7]

Three types of controllers, proportional, integral and derivative (PID) were developed in 1930 s, still the most widely applied industrial controllers. This succeed is a result of many good features of this algorithm such as simplicity, robustness and wide applicability [8]. Transfer function $\left(G_{C}\right)$ of PID Controller generally expressed as

$$
G_{C}(s)=K_{C}\left(1+\frac{T_{I}}{s}+T_{D} s\right)
$$

where $K_{C}$, is proportional gain $T_{I}$ is integral time and $T_{D}$ is derivative time [8, 9]. Many different tuning methods have been proposed from 1942 to the present day for gaining better and more acceptable control system response based on desirable control objectives such as percent of overshoot, offset, settling time, manipulated variable behavior etc. PID controller tuning methods are classified into closed loop methods such as Ziegler-Nichols method, Tyreus-Luyben method and Damped Oscillation method etc., and Open loop methods like open loop Ziegler Nichols methods, Cohen and Coon method, Minimum error criteria methods etc. $[8,10]$

Nevertheless, there exist very few literatures [11 - 13] about PID tuning of flow control system, in this study, attention paid mainly to evaluate the parameters of PID controller applied for turbine flow control of water assembled in a table top process control trainer 
(PCT) system. Since the Ziegler-Nichols models are simple and most popular in industrial applications, closed loop Ziegler-Nichols tuning method was adopted to estimate PID controller parameters. Tuned variables were set for proportional (P), proportional integral (PI), and proportional integral derivative (PID) control mechanisms and several experiments were performed at different set values to identify the system overall performance.

\section{Closed Loop Ziegler-Nichols Tuning Method}

This is a heuristic trial and error tuning process based on sustained oscillations that was proposed by Ziegler and Nichols in 1942. This is probably the most widely used method for tuning of PID controllers, is also known as online or continuous cycling or ultimate gain tuning method $[8,14]$.

Table 1

Closed loop calculations PID controller parameters, $K_{C}, T_{I}$ and $T_{D}$

\begin{tabular}{cccc}
\hline Controller/Parameter & $\begin{array}{c}\text { Proportional Gain } \\
\left(K_{C}\right)\end{array}$ & Integral Time $\left(T_{I}\right)$ & $\begin{array}{c}\text { Derivative Time } \\
\left(T_{D}\right)\end{array}$ \\
\hline $\mathrm{P}$ & $0.5 K_{c r}$ & $\infty$ & 0 \\
$\mathrm{PI}$ & $0.45 K_{c r}$ & $P_{c r} / 1.2$ & 0 \\
$\mathrm{PID}$ & $0.6 K_{c r}$ & $0.5 P_{c r}$ & $0.125 P_{c r}$ \\
\hline
\end{tabular}

It applies only proportional gain $\left(K_{P}\right)$ in a feedback control loop by neglecting integral and derivate actions.

The process usually begin with low values of gain, $K_{P}$ followed by gradual increase until a steady state oscillation occurs.

The gain corresponding to sustained oscillation period, $P_{c r}$ can be evaluated as $K_{c r}=100 / P_{b}$ , where $P_{b}$ is the proportional band of the system. After that PID controller parameters can be calculated as in Table 1. [15 - 17].

\section{Materials \& Methodology}

Materials: Ambient air passed through compressor followed by oil catch filter was used as fluid to provide pressure for differential pressure flow sensor and also to serve in I to $\mathrm{P}$ Converter.

Water fetched from municipal water supply lines of Mai-Nefhi was utilized as process fluid.

\section{Experimental Setup:}

A table top process control trainer (PCT) system, originally supplied by Matrix Global Pvt. Ltd. India but accessed from the Process Control lab, Department of Chemical Engineering, Mainefhi College of Engineering \& Technology (MCOETEC). Water was taken as process fluids, and the air served as utility in differential pressure flow sensor.

PCT mainly composed of a sump tank of 70 liters, mounted horizontally, $1 / 2$ HP centrifugal pump to drive water from sump tank to the process, a compressor that compress air up to 10 bar with automatic on/off operation, an oil catch filter to purify air that comes into the process, a process tank of 70 liter mounted vertically, control valve and computer loaded with PID controller software.

All these hardware elements were connected with auxiliary fittings such as pipelines, pressure dials, inline valves and transmission lines as shown in Figure 1. 


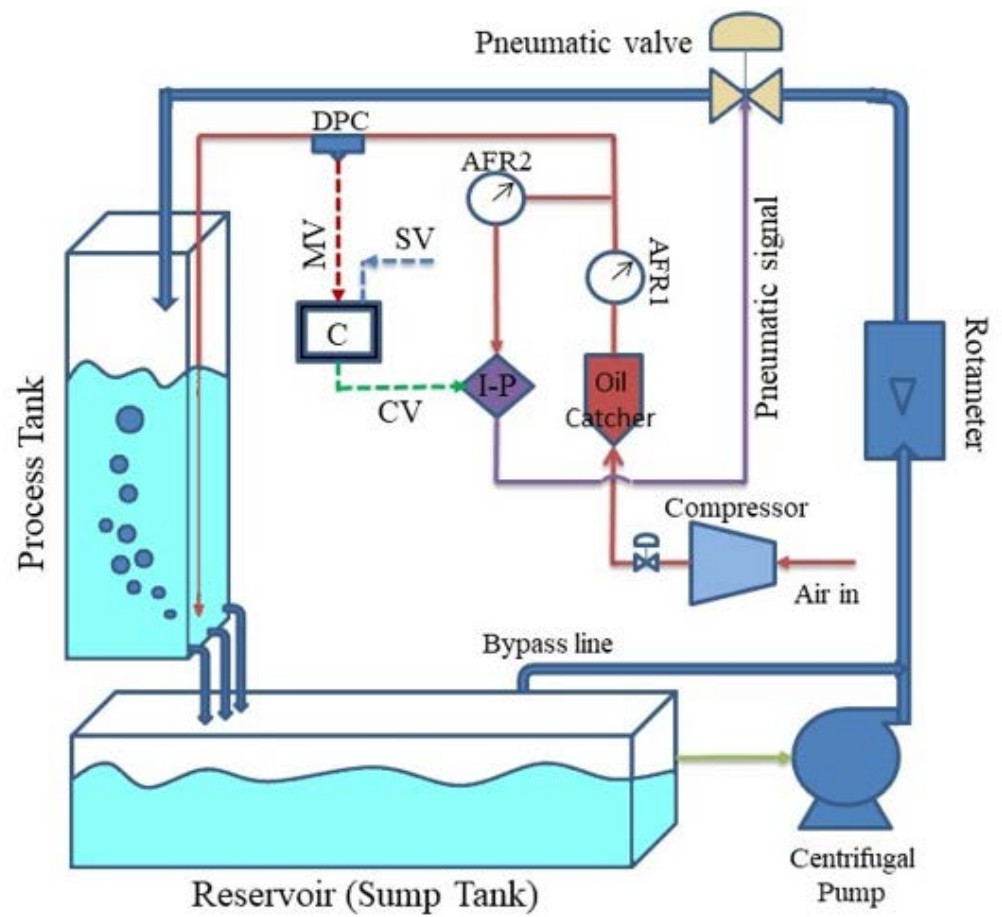

Figure 1. Schematic diagram of process control trainer (PCT) system.

\section{Methodology}

To configure flow control system in to a closed loop control, the connections were made according to the wiring sequence shown in the text box below prior to conduct experiments. As a primary phase, a set of experiments were performed to evaluate PID controller parameters by using Ziegler-Nichols method.

\section{Tuning of PID controller of Water flow control system:}

According to Ziegler Nichol's Second method, a closed loop setup was arranged as shown in Figure 2 through accomplished wiring sequence. Initially the system intake pressure was arranged at 1 bar by watching on pressure dial AFR1. Prior to switch on the pump, all the necessary parameters as shown in Figures $3 \& 4$ were set on main window and on settings window of PID controller software installed on PC. As the pump capacity is quite high, a bypass line was provided, and the inflow valve was kept open, and also two of the exit valves of process tank were also kept open. The pump made on and for a set value of $30 \%$, the responses of the system in Figure 1 were noted by changing proportional band $\left(P_{b}\right)$ values in ascending order, from 3 to 11 manually in the main window of PID Controller application until a sustained wave with constant amplitude was recorded. The data generated from the different sets of experiments were collected and analyzed clearly to identify a perfect wave with a constant amplitude.

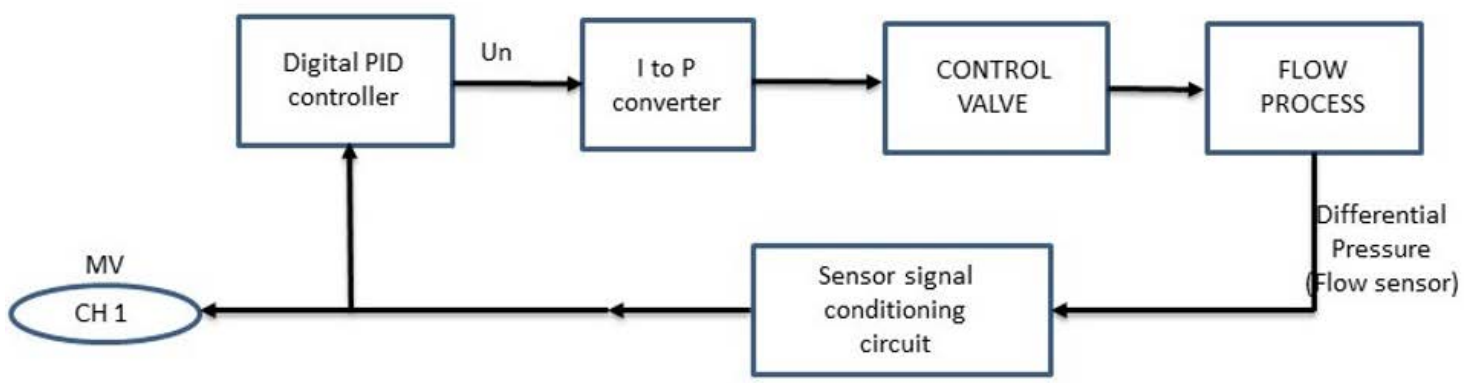

Figure 2. Block diagram of closed loop control of flow control. 


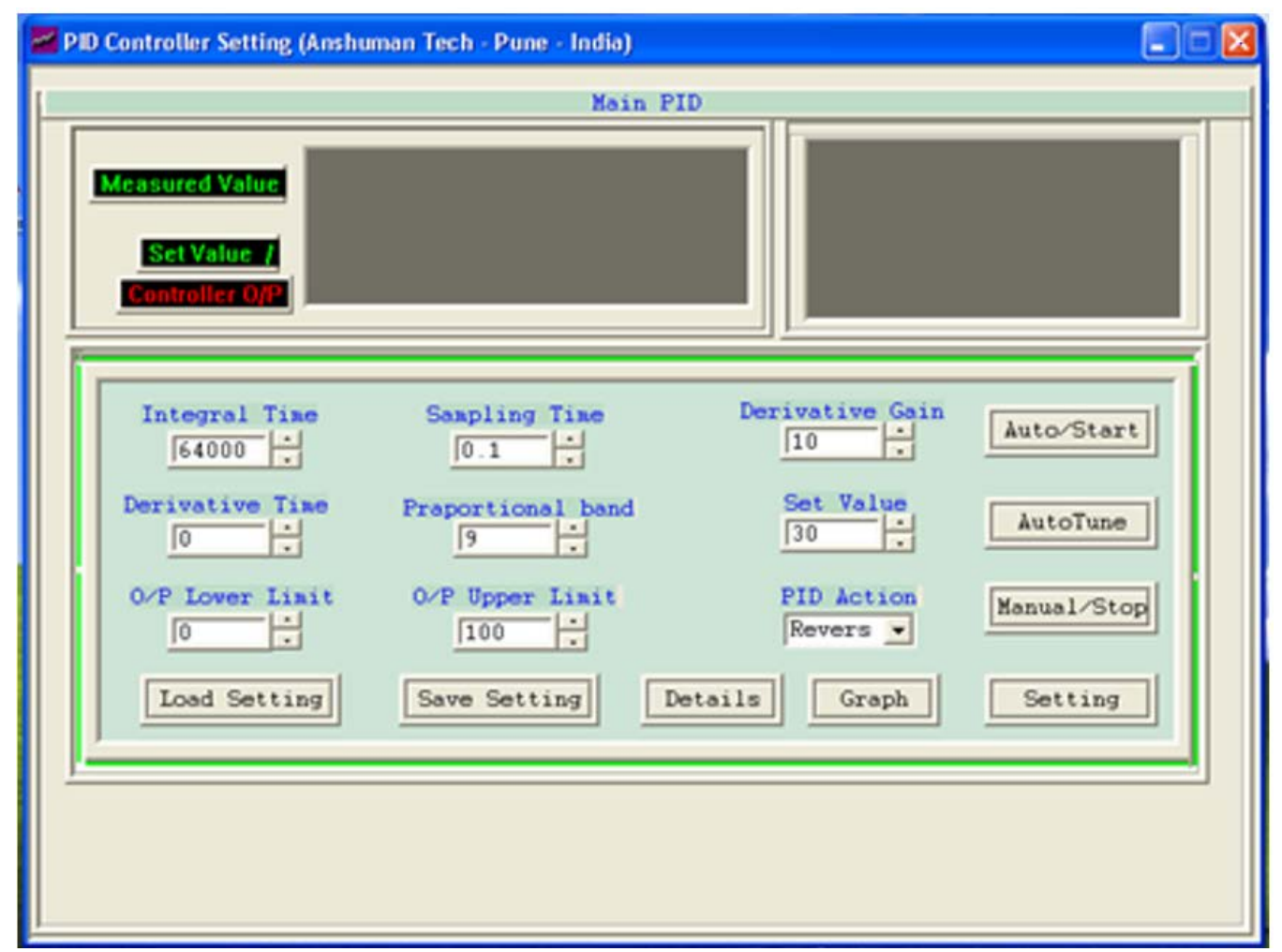

Figure 3. Parameters Set on Main window of PID controller software.

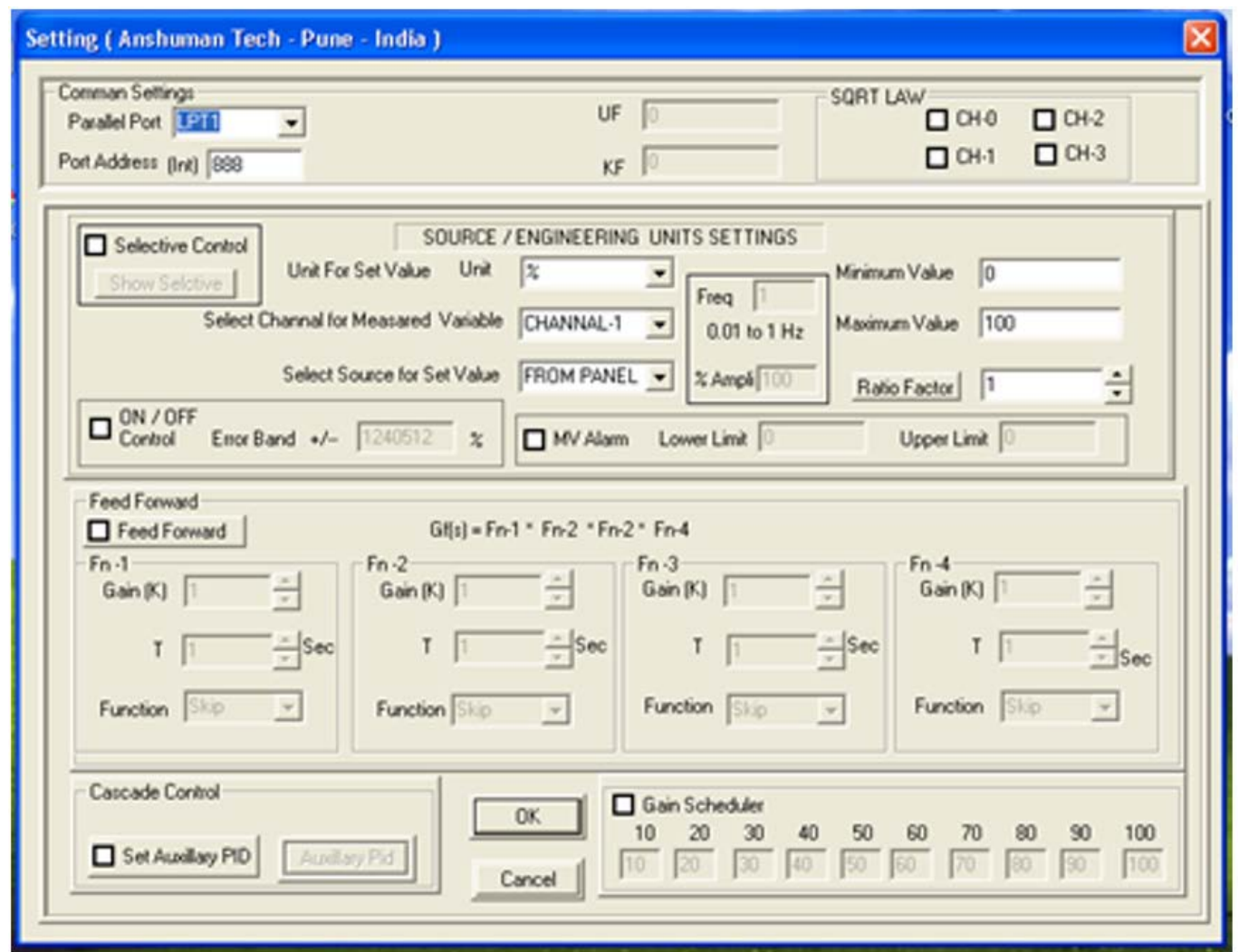

Figure 4. Parameters Set on Setting window of PID controller application.

Wiring Sequence: Pump - L14 on EMT8, Pump - N15 on EMT8, FLOW O/P (14 of Signal conditioning panel) - Computer interface panel (CIP) CH1, CIP 6 - CIP 9, CIP 10 - (+ve) of I to $P$ converter, (-ve) of I to P converter - CIP 20, FLOW O/P (14 of SCP) - (+ve) of DPM, (-ve) of DPM - GND on SCP. 


\section{Performance Studies on Step responses of water flow control system}

Estimated Ziegler-Nichols parameters of P, PI and PID controllers were employed on physical system through PID controller application and a range of step values starting from $20 \%$ to $50 \%$ were applied to identify the system response. Produced data with a sampling time of $0.1 \mathrm{sec}$ from all the experiments were examined independently for the comparison of variables such as overshoot, decay ration, settling time and offset.

\section{Results \& Discussion}

To evaluate Ziegler-Nichols tuning parameters of single loop flow control system, experiments were conducted on a closed loop system with $\mathrm{P}$ controller by changing proportional band $\left(P_{b}\right)$ gradually from 3 to 11 for a set value of $30 \%$ of flow rate and their responses were plotted as shown in Figures $5 \& 6$. Response of the system in the wave form were notified at lower $P_{b}$ values and wave structured response was totally disappeared at $P_{b}=11$ as shown in Figure 7. Most of the cases, total number of samples in a cycle were equal but the position of amplitude was shifted to the right, counted 8 samples in the left and 6 on the right as shown in figures $5 \& 6$. But a constant value of amplitude measured exactly at middle of the cycle was clearly seen with equally distributed samples in case of experiments at proportional band $\left(P_{b}\right)$ of 9 and its corresponding gain was evaluated as $K_{c r}=$ $100 / P_{b}$. Measured value (MV) verses time $(\mathrm{t})$ was plotted and the period of oscillation $\left(P_{c r}\right)$ was calculated as

$$
\begin{gathered}
P_{c r}=\text { Number of Samples in a cycle }(N) \times \text { Sampling time }\left(t_{s}\right) \\
P_{c r}=14 \times 0.1=1.4 \mathrm{sec}
\end{gathered}
$$

Other tuning parameters of P, PI and PID controllers were estimated as given by Table 2 .

Table 2

\section{Estimated Ziegler-Nichols tuning parameters of $P, P I$ and PID controllers}

\begin{tabular}{ccccc}
\hline Controller/Parameter & $\begin{array}{c}\text { Proportional } \\
\text { Gain }\left(K_{P}\right)\end{array}$ & $\begin{array}{c}\text { Proportional } \\
\text { Band }\left(P_{b}\right)\end{array}$ & $\begin{array}{c}\text { Integral Time } \\
\left(T_{I}\right)\end{array}$ & $\begin{array}{c}\text { Derivative } \\
\text { Time }\left(T_{D}\right)\end{array}$ \\
\hline P & 5.55 & 18 & 64000 & 0 \\
PI & 4.99 & 20 & 1.167 & 0 \\
PID & 6.67 & 15 & 0.7 & 0.175 \\
\hline
\end{tabular}

\section{Effect of Tuned Parameters on Step Response of the System}

Performance studies on water flow control system at different step shifts, $20 \%, 30 \%$, $40 \%$ and $50 \%$ were conducted with Ziegler-Nichols tuning variables of P, PI and PID controllers and corresponding data was generated with a sampling time of $0.1 \mathrm{sec}$. It was noticed that there wasn't further change in the output for set values higher than $50 \%$. In fact, the system could able to attain the maximum flow rates of $40.39 \%, 40.78 \%$ and $41.17 \%$ with P, PI and PID control mechanisms respectively for $50 \%$ of set value as shown in Table 3. It was clearly observed that Pi and PID controllers eliminated offset at lower flow rates as set values and the system could able to attain a maximum flow rate of $82.34 \mathrm{LPH}$ though the set values were taken to further higher. Set values (SV) and measured values (MV) were opted as ordinates and time $(\mathrm{t})$ taken as abscissa and their resultant responses were plotted for $\mathrm{P}$ (figure 8), PI (figure 9) and PID (figure 10) controllers to analyze the system characteristics. 
Table 3

Ultimate flow rates attained by P, PI and PID Controllers for different set values

\begin{tabular}{cccccccccccccc}
\hline $\begin{array}{c}\text { Set value } \\
\text { (SV) }\end{array}$ & & $20 \%$ & \multicolumn{1}{c}{$30 \%$} & \multicolumn{3}{c}{$40 \%$} & \multicolumn{3}{c}{$50 \%$} \\
\hline $\begin{array}{c}\text { Controller } \\
\text { P }\end{array}$ & PI & PID & P & PI & PID & P & PI & PID & P & PI & PID \\
\hline $\begin{array}{c}\text { Flow rate } \\
\text { in \% (MV) }\end{array}$ & 18.82 & 20 & 20 & 25.49 & 29.8 & 29.8 & 34.5 & 39.61 & 39.61 & 40.39 & 40.78 & 41.17 \\
$\begin{array}{c}\text { Flow rate } \\
\text { in LPH }\end{array}$ & 37.64 & 40 & 40 & 50.98 & 59.6 & 59.6 & 69 & 79.22 & 79.22 & 80.78 & 81.56 & 82.34 \\
Offset & 1.18 & 0 & 0 & 4.51 & 0.2 & 0.2 & 5.5 & 0.39 & 0.39 & 9.61 & 9.22 & 8.83 \\
\hline
\end{tabular}

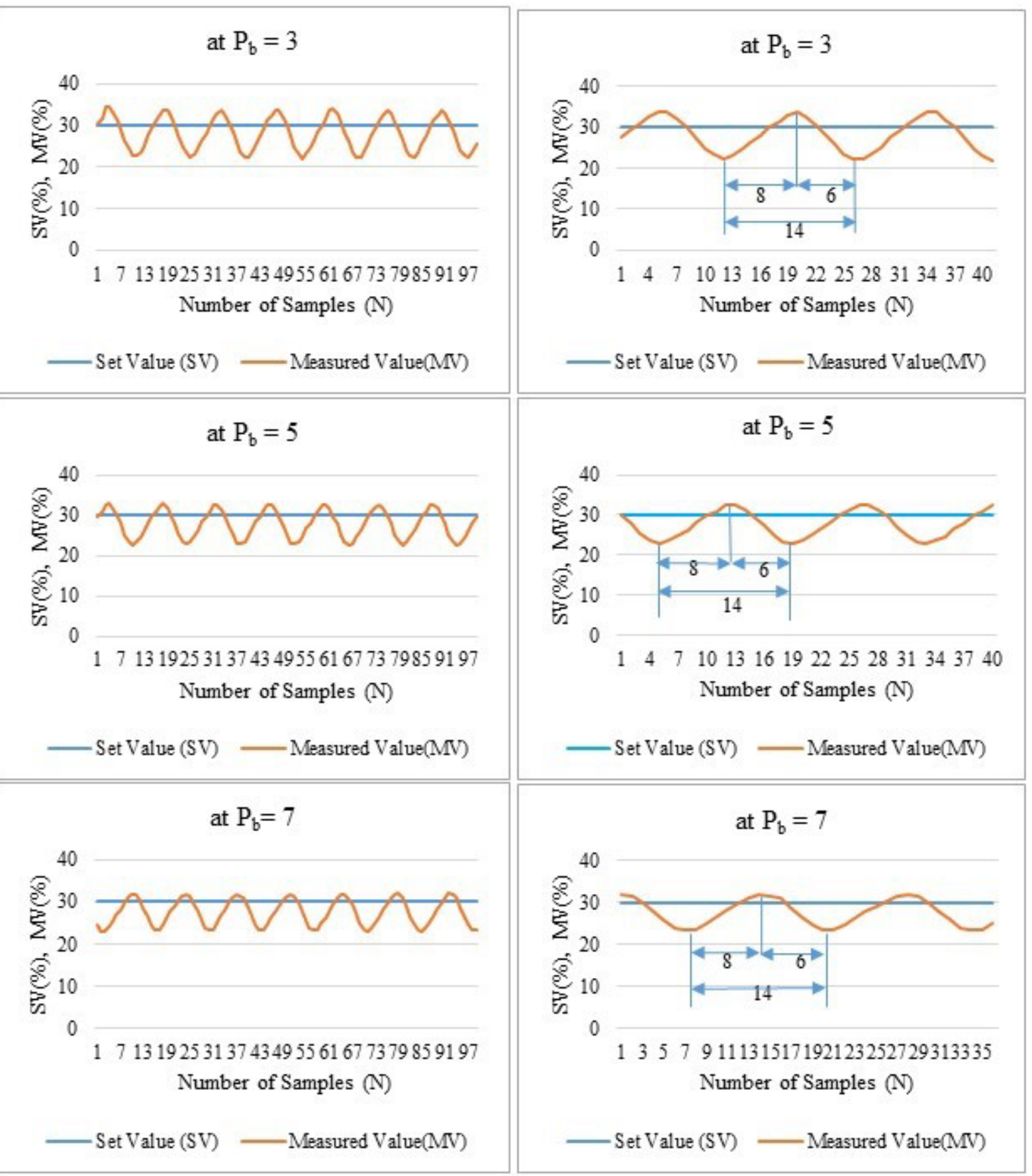

Figure 5. Response of P controller of water flow system for $30 \%$ of Set Value at different $P_{b}$ values of 3,5 and 7 . 

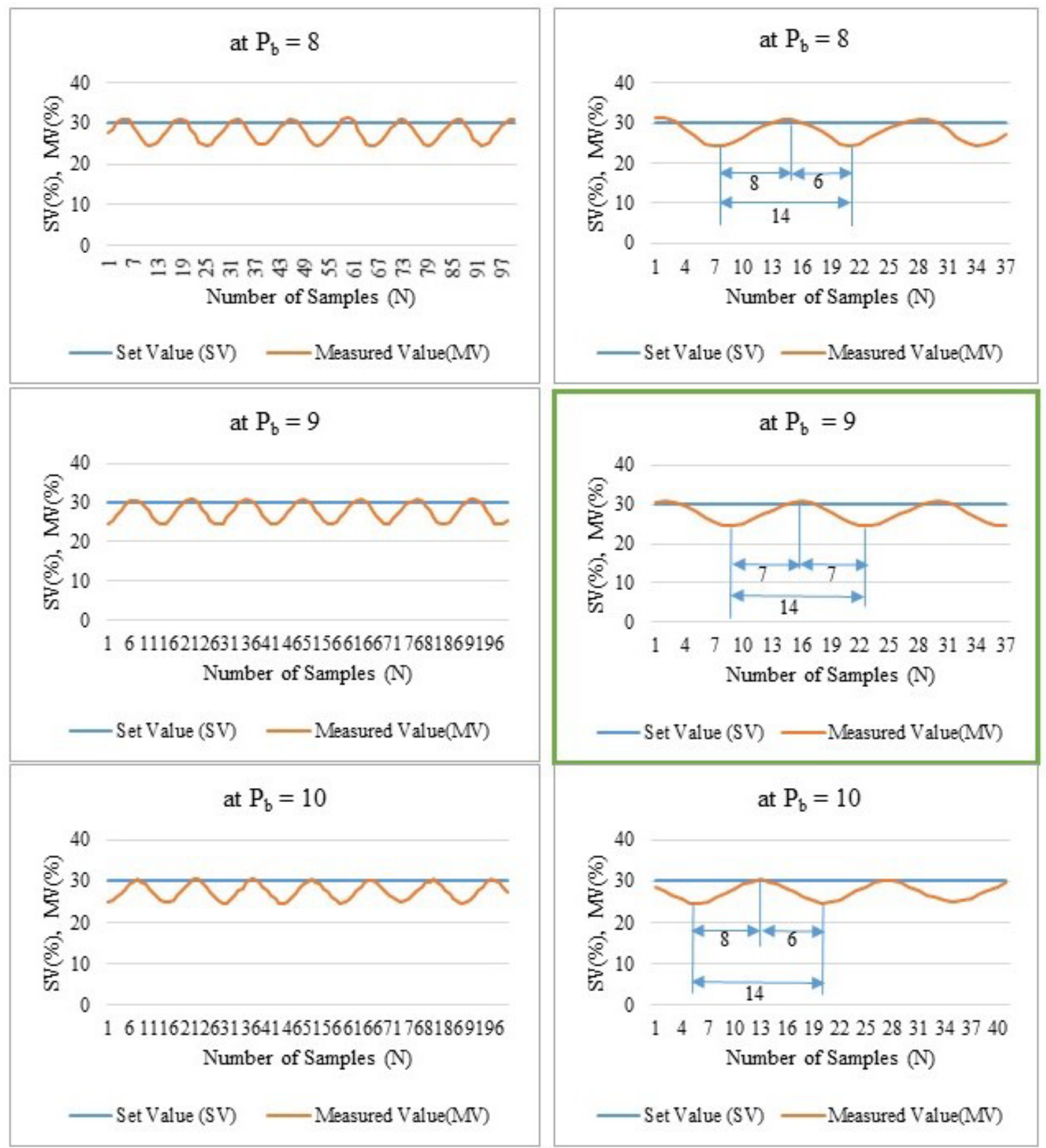

Figure 6. Response of $\mathrm{P}$ controller of water flow system for $30 \%$ of Set Value at different $P_{b}$ values of 8,9 and 10 .
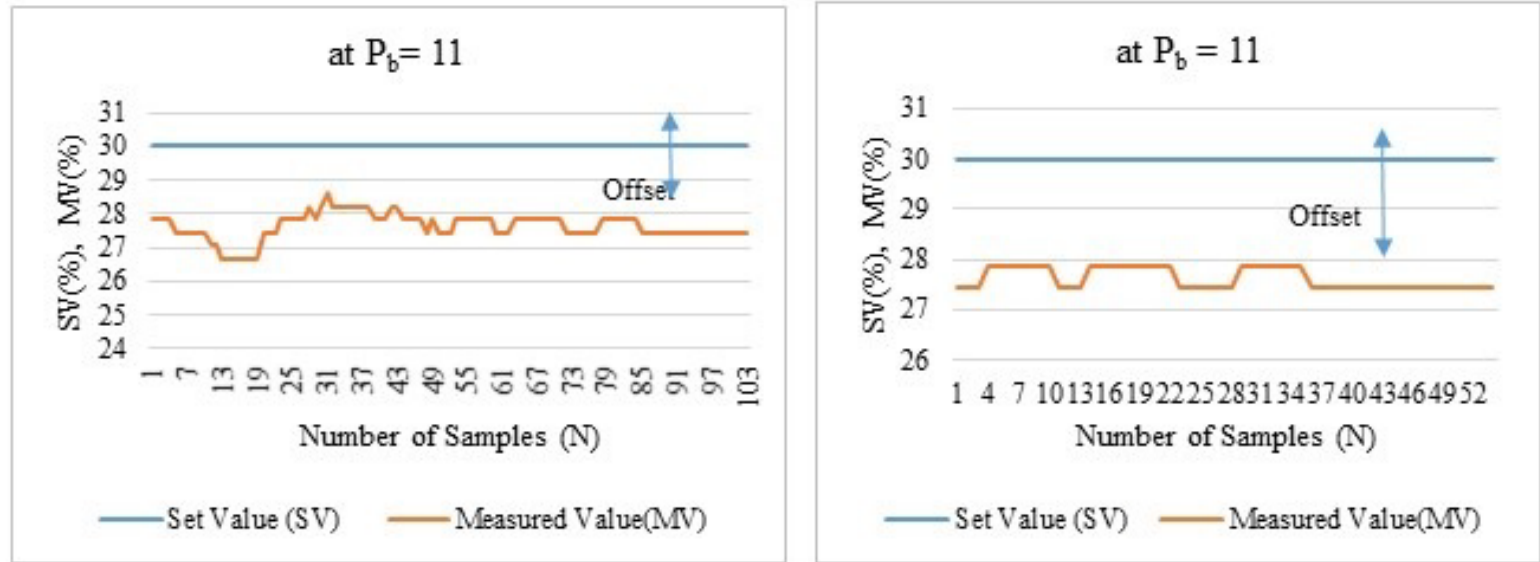

Figure 7. Step response of closed loop control of water flow system for $30 \%$ set value at $P_{b}=11$. 
P Controller

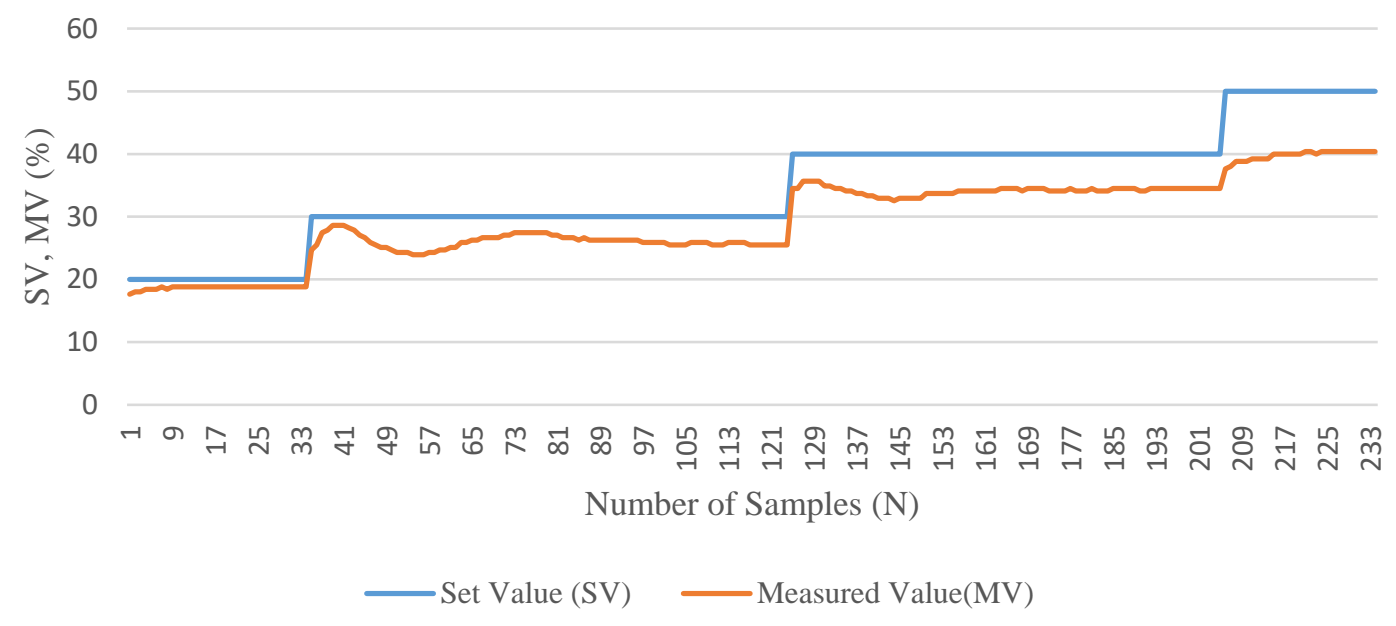

Figure 8. Step responses of water flow control system with $\mathrm{P}$ controller.

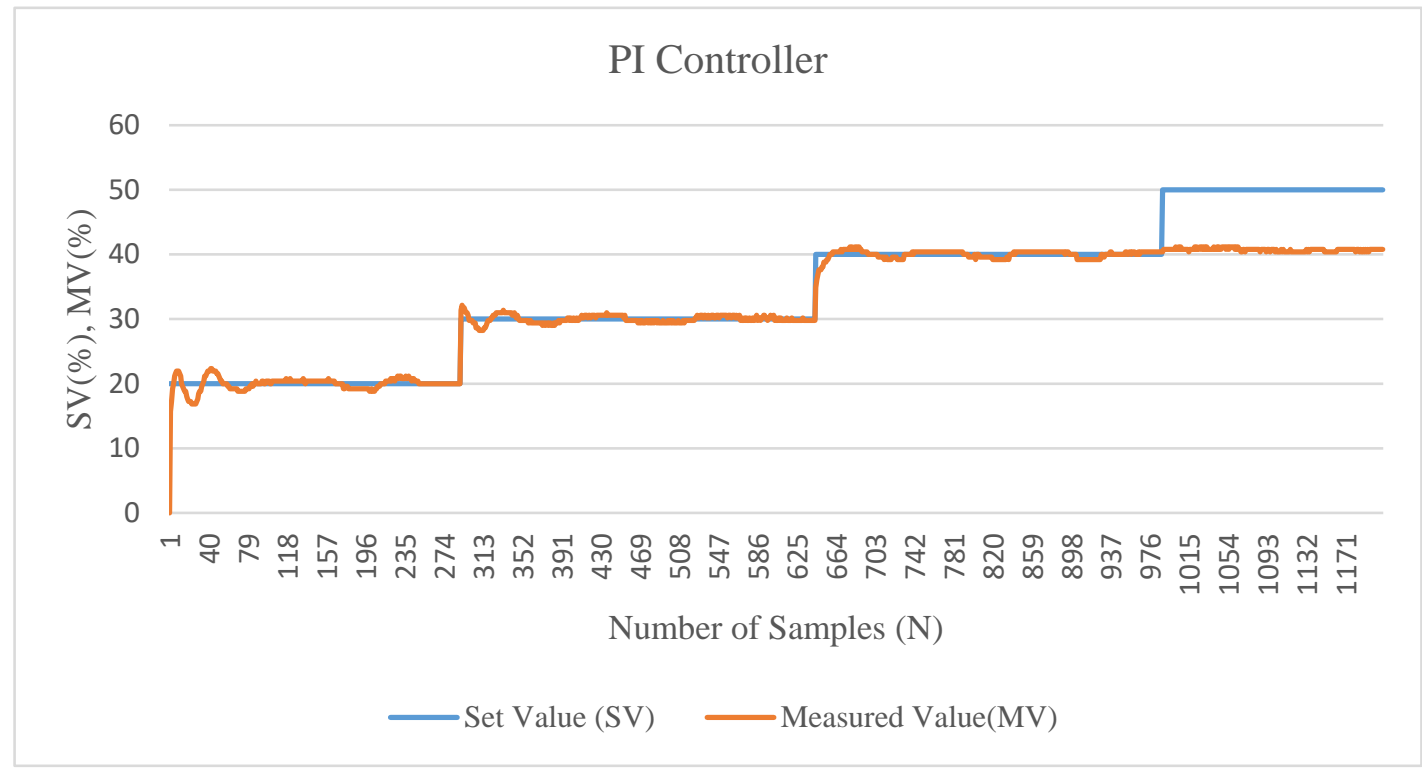

Figure 9. Step responses of water flow control system with PI controller.

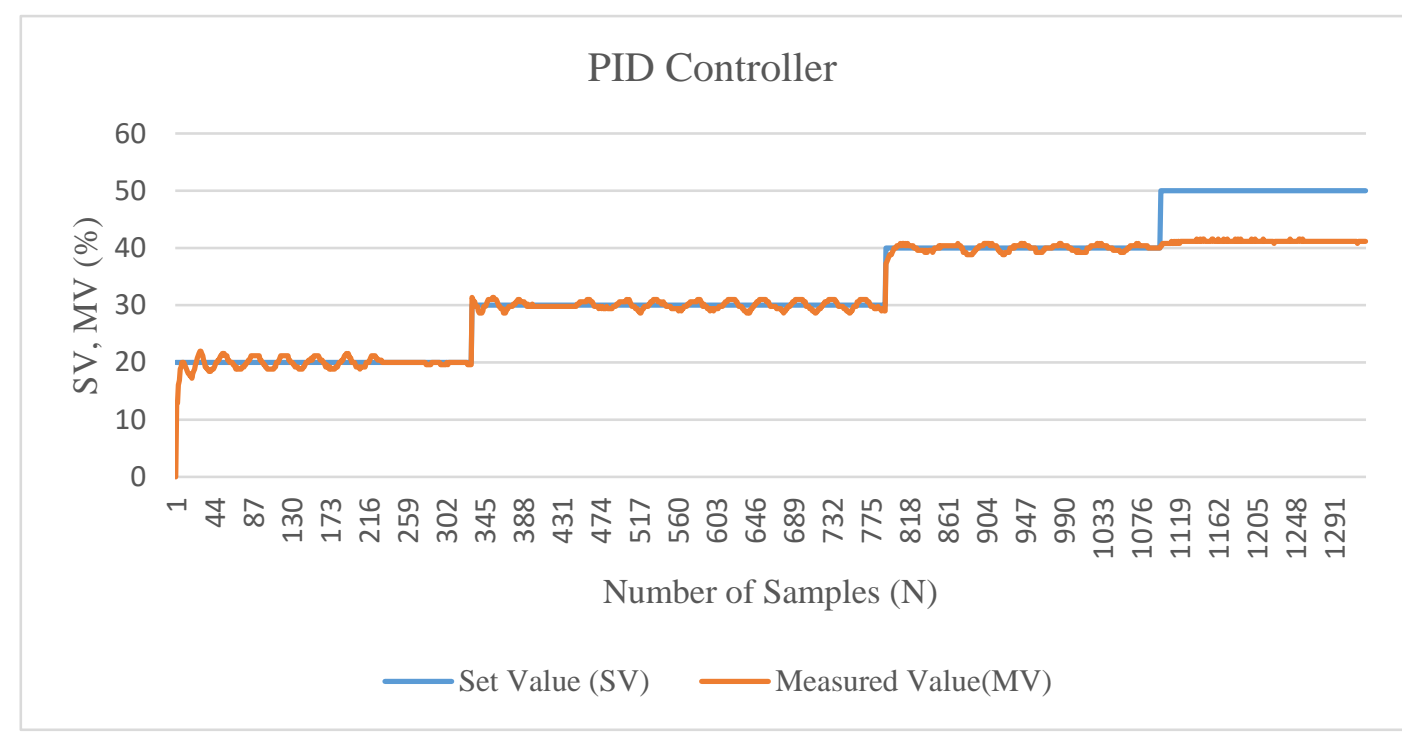

Figure 10. Step responses of water flow control system with PID controller. 


\section{Characterization of P, PI and PID controllers of Water flow control system}

For different step disturbances, the system response plots were developed to evaluate the characteristic parameters of P, PI and PID control mechanisms. Overshoot, Decay ratio, settling time and Offset values were calculated and the results are furnished in Table 4. When the system facilitated exclusively only $\mathrm{P}$ control through setting estimated proportional band value of 18 , and applied different step changes, system performed with faster settling times than PI and PID controllers, especially at $20 \%$ and $50 \%$ set values as shown in Figure 11 whereas PI controller of tuned parameters, has recorded with higher settling times than other two modes. In other words, $\mathrm{P}$ controller has led to consistent steady state errors which is called as offset.

Table 4

Estimated characteristic parameters of step response of water flow control system with $P, P I$ and PID control mechanisms

\begin{tabular}{cccccccccccccc}
\hline $\begin{array}{c}\text { Set value } \\
\text { (SV) }\end{array}$ & & $20 \%$ & \multicolumn{3}{c}{$30 \%$} & & \multicolumn{3}{c}{$40 \%$} & \multicolumn{3}{c}{$50 \%$} \\
\hline Controller & P & PI & PID & P & PI & PID & P & PI & PID & P & PI & PID \\
\hline $\begin{array}{c}\text { Overshoot } \\
\begin{array}{c}\text { Decay } \\
\text { Ratio }\end{array}\end{array}$ & NA & 11.75 & 9.8 & NA & 3.27 & 4.57 & NA & 1.95 & 1.95 & NA & 0 & 0 \\
$\begin{array}{c}\text { Settling } \\
\text { time }\end{array}$ & 9 & 250 & 232 & 83 & 326 & 69 & 68 & 324 & 62 & 18 & 175 & 23 \\
Offset & 1.18 & 0 & 0 & 4.51 & 0.2 & 0.2 & 5.5 & 0.39 & 0.39 & 9.61 & 9.22 & 8.83 \\
\hline
\end{tabular}

With increasing step changes, offset values also increased and recorded higher values for P controllers than PI and PID controllers as depicted in Figure 12. As the purpose of integral action is to provide lower or zero offset value, it was observed that the PI controllers holds zero offset at $20 \%$ step disturbance, and very close to zero at 30 and $40 \%$ step changes but it is high at $50 \%$ step change because the physical design of the specific system was identified as it doesn't support flow rates greater than $41.56 \%$ of its capacity.

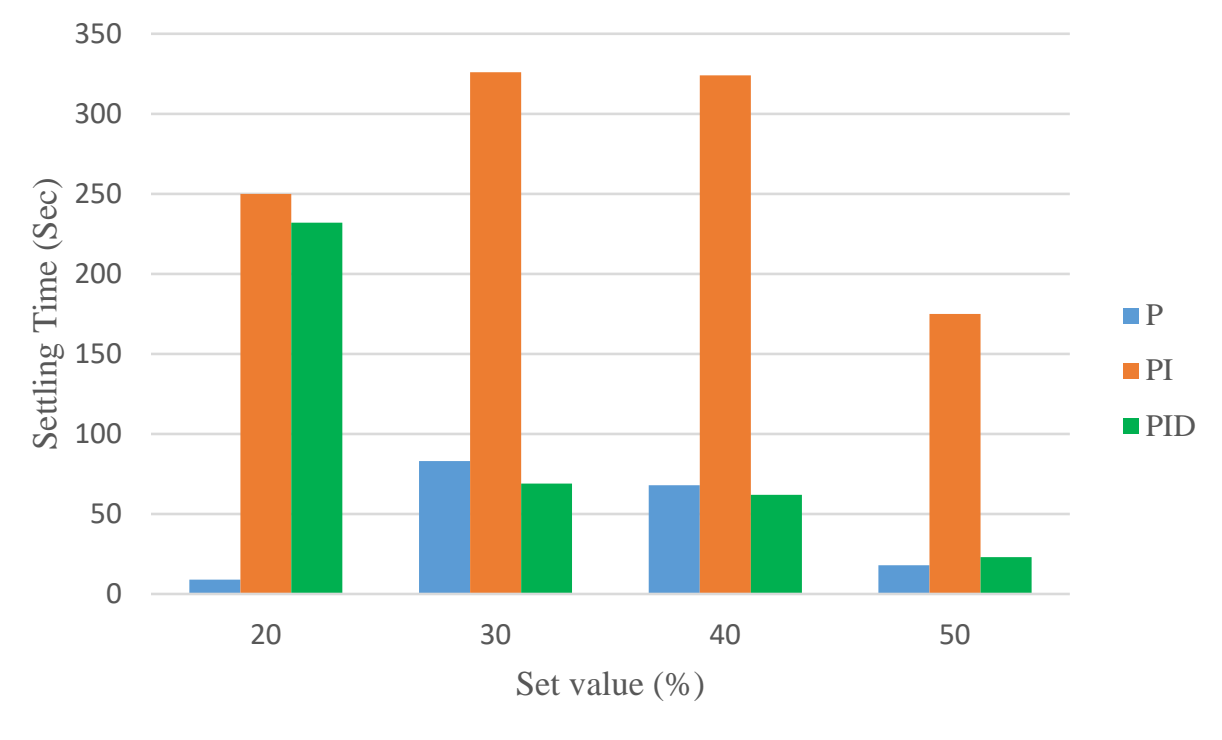

Figure 11. Settling times of P, PI and PID controllers at different set values. 
Overshoot is defined as the ratio of amplitude of the peak to the set value and the ratio of two successive amplitudes is known as decay ratio $[18,19]$. Overshoot and decay ratios of PI and PID controllers were compared in figures 13 and 14, and notified the values are significant for step changes of 20,30 and $40 \%$ but not at $50 \%$ as it beyond the range of the system. PI controller has recorded higher overshoots than PID with lower decay ratios. As the integral term integrates or continually sums up error over time even a small error amount of persistent error calculated in the process will aggregate to a considerable amount of over time [20], therefore higher response times were observed for PI Controllers.

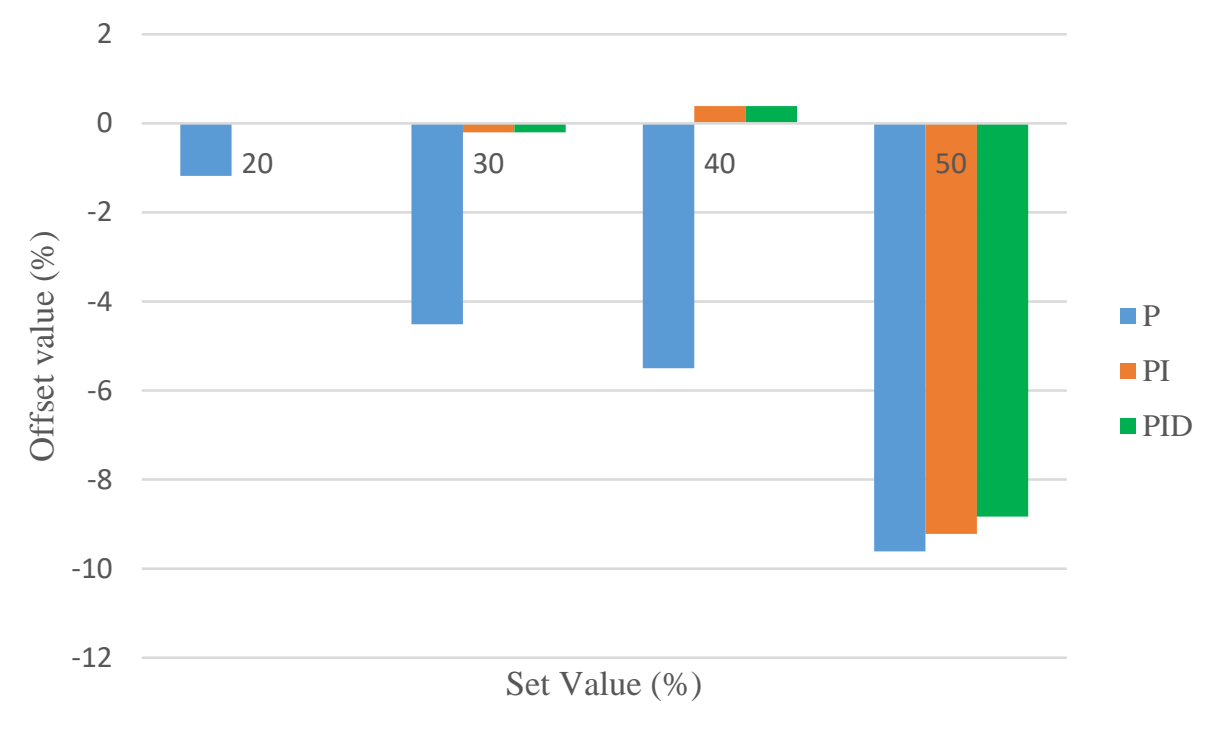

Figure 12. Offset values recorded for P, PI and PID controller at different set values

The configuration of PID control has used full set of tuned parameters, proportional, integral and derivative variables and it exhibited aggressive response with lower overshoot values but it increased chatter on the control output signal. Thus, PID control is preferable for use in steady state processes or processes that either respond slowly or have little-to-no noise [20]. Flow control in general possess low sampling time between 1 - $5 \mathrm{sec}$ [20], therefore because of faster and stable responses, $P$ controller could be a better choice for water flow control if offset to be zero is not a constraint. Else, PI controller would provide zero offset although it consumes longer time to settle with moderate maintenance.

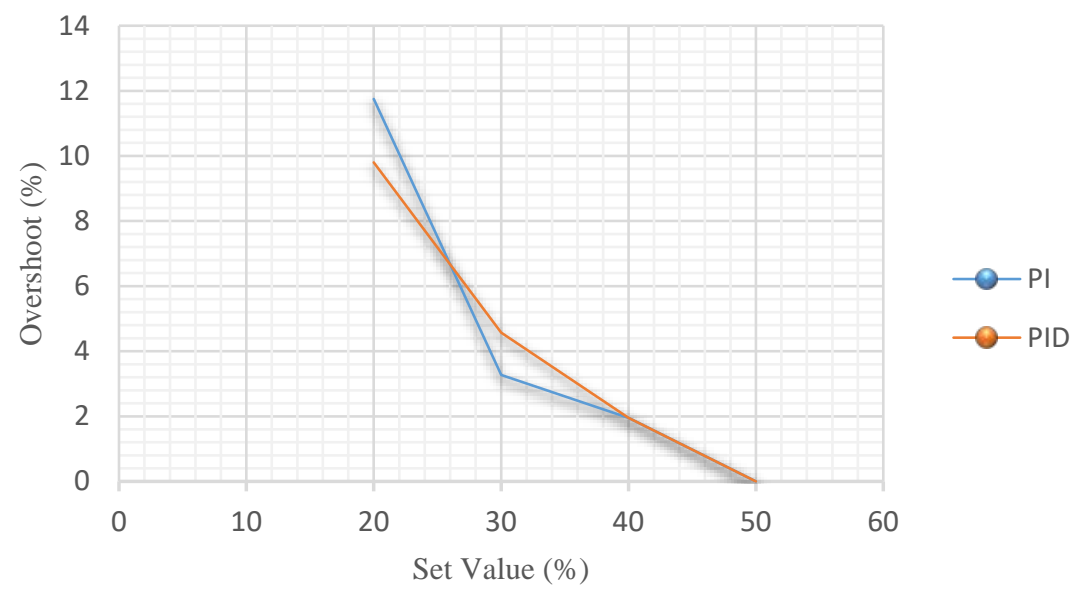

Figure 13. Comparison of overshoot values of PI and PID controllers. 


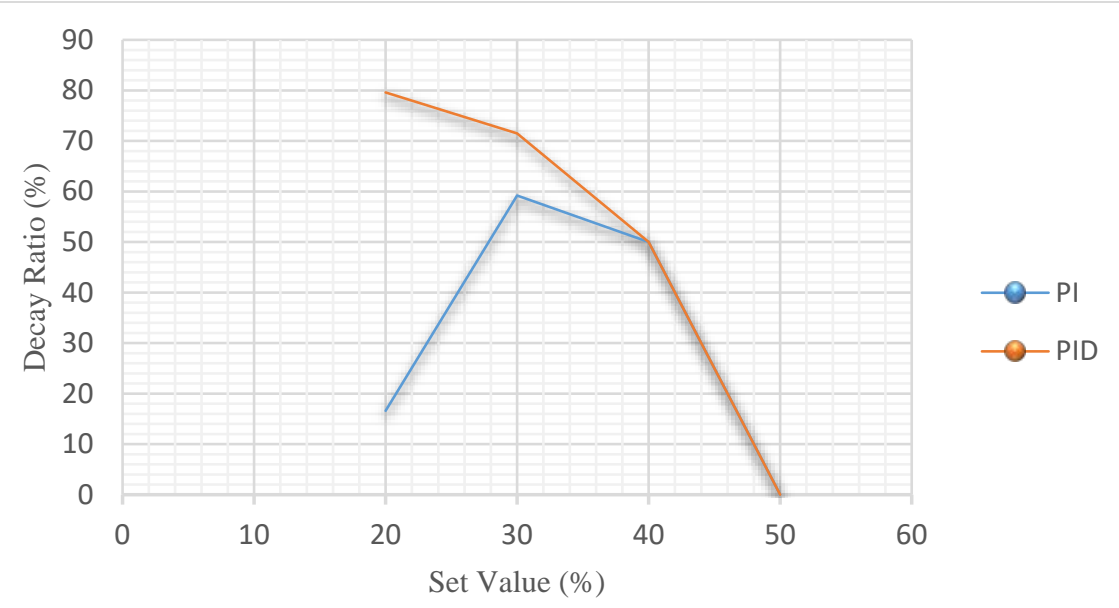

Figure 14. Comparison of decay ratios of PI and PID controllers.

\section{Conclusions}

As Eritrea is striving to manage its limited water resources, application of flow controllers to improve water distribution system in the country addressed for the first time in this work. A table top process control trainer (PCT) was tested through proportional(P), integral (I) and derivative (D) control mechanisms. Applying exclusively $\mathrm{P}$ control action, critical period of oscillation $\left(P_{c r}\right)$ was estimated as $1.4 \mathrm{sec}$ at proportional band value of 9 . Tuning parameters of PID controller for water flow control system were evaluated using Closed loop Ziegler-Nichols method. Setting sample time at $0.1 \mathrm{sec}, \mathrm{P}, \mathrm{PI}$ and PID controller performance studies were conducted with tuned variables on the water flow control system at different step disturbances between $20-50 \%$ and their corresponding responses were characterized. $\mathrm{P}$ controller exhibited faster responses with consistent increments in offset, $\mathrm{PI}$ controller recorded highest overshoot values with negligible offset and prolonged settling times. PID controller showed less overshoot values and faster response times than PI but it increased chatter on the control output signal. The study revealed that the system could attain maximum controlled flow rates of 83.12 LPH (Litre Per Hour) with a set value of 50\%, and it retained there for further higher set values. Therefore, the system can be safely controlled between 0-80 LPH. Since flow control systems possess shorter sampling times between 1-5 sec, if the offset is not a major constraint, $P$ controller could be reflected suitable with simple design and minimum expenditure, else PI controller makes offset to zero though it possesses higher settling times. In other words, PID controller is complex using more tuning parameters, need expensive maintenance, and has resulted an intermittent noise in the output signal.

Acknowledgements: Authors would like to express their deepest gratitude to HOD, Department of Chemical Engineering Mr. Abraham Yohannes, for his untiring endeavour in establishing Chemical Engineering laboratories at Mai Nefhi College of Engineering and Technology (MCoET). Authors also want to convey their thankfulness to Dr. Sujana Ramesh for her consistent patronage in proof reading and editing the document. They enhance their thankfulness to Staff of Process Control laboratory, and to all the Faculty and technical staff of the Chemical engineering, MCoET for their persistent support. Authors would like to extend their inmost gratefulness to the Dean of MCoET, Dr. Kahsay Neguse for his diligent effort to encourage earnest research from the college. 


\section{References}

1. Grace Kam Chun Ding. Wastewater Treatment and Reused -The Future Source of Water Supply. In: Encyclopedia of Sustainable Technologies, 2017, vol 4, pp. 43 - 52. http://dx.doi.org/10.1016/B978-0-12409548-9.10170-8

2. A. Ramesh babu, Samsom K., Samuel M. Experimental Studies on Step Response of Water Level Control System with P, PI and PID Control Mechanisms, In: International Research Journal of Engineering and Technology (IRJET), 2020, 10(7), pp. 1504 - 1509.

3. Ranade V.V., Bhandari V.M. Industrial Wastewater Treatment, Recycling and Reuse. Amsterdam: Elsevier Ltd, $1^{\text {st }}$ ed, 2014.

4. UNESCO, 2015. The United Nations World Water Development Report: water for a sustainable world. United Nations Educational, Science and Cultural Organization, France.

5. UNICEF, WASH/FN/27/2019 Prioritizing Climate Resilience, Sustainability and Equity: OneWASH Programming in Eritrea. In: UNICEF's WASH field note. Available online

https://www.researchgate.net/profile/David-

Tsetse/publication/338710232_Prioritizing_Climate_Resilience_Sustainability_and_Equity_-

_One_WASH_programming_in_Eritrea/links/5e2653d692851c89c9b5a584/Prioritizing-Climate-Resilience-

Sustainability-and-Equity-One-WASH-programming-in-Eritrea.pdf

6. Technology Zones. How Important is Flow Control in Hazardous Environments, In: Fluid Control Ltd articles, 2019. Available at the following weblink.

https://fluidhandlingpro.com/how-important-is-flow-control-in-hazardous-

environments/\#: :text=Flow\%20controllers\%20are\%20designed\%20to,they\%20are\%20being\%20used\%20i $\mathrm{n}$.

7. Fujikin Corp Group, Flow Control Systems, Catalogue No.712-01E-H, 2013.

8. Mohammad S., Alireza Z. Comparison of PID Controller Tuning Methods, In: Computer Science, 2012. Online Source to access

http://ie.itcr.ac.cr/einteriano/control/clase/Zomorrodi_Shahrokhi_PID_Tunning_Comparisn.pdf

9. Thomas E. Marlin. Process Control-Design processes and Control systems for Dynamic response, New York: McGraw Hill, Inc. $2^{\text {nd }}$ ed, 2000.

10.P.Sathishkumar, N. Selvaganesan. Tuning of Complex Coefficient PI/PD/PID controllers for a universal plant structure, In: International Jurnal of Control, 2020, https://doi.org/10.1080/00207179.2020.1755726

11. Philip R. Page., Enrico Creaco. Comparison of Flow Dependent Controllers for Remote Real-Time Pressure control in Water Distribution System with Stochastic Consumption, In: Water, MDPI, 2019, 11, pp. 422 doi:10.3390/w11030422

12. Sina R., Cristobal V.J., Raheleh J., Alexander G. Flow Control of Fluid in Pipelines Using PID controller, In: IEEE Access, 2019, 7, pp. 25673 - 25680. DOI 10.1109/ACCESS.2019.2897992

13. Hakki U.U., Altug. I. Performance and Robustness of Flow Controllers Designed Using Non-Casual Uncertainty Blocks, In: Asian Journal of Control, Wiley Online Library, 2013, 15(1), pp. 214 - 224. doi: 10.1002 /asjc.488

14. Jietae Lee. On-line PID controller tuning from a single, closed-loop test, In: AIChE Journal, 1989, $35(2)$, pp.329 https://doi.org/10.1002/aic.690350221

15. Matrix Global Private Limited. User's Guide of Process Control Trainer (PCT), Noida: Matrix Global Pvt.Ltd., version 1.5, 2009.

16. Robert Ziff. Chemical Process Dynamics and Control, Book-I, In: University of Michigan, 2007, pp. $693-694$

17. Stephanopolous G. Chemical Process Control, New Jersey: Prentice-Hall Inc., 1984, pp, 352 - 354

18. Donald.R. Coughanowr. Process Systems Analysis and Control, New York: McGraw Hill Publications, 2nd ed, 1991, pp. 96 - 98

19.D.E.Seborg, T.F.Edgar,D.A. Mellichampt. Process Dynamics and Control, New Jersey: John Wiley \& Sons Inc., 2004, $2^{\text {nd }} E d$.

20. Robert.C.Rice. PID Tuning Guide-Yokogawa,Control station Inc., 2010, pp. 20 - 23.

https://web-material3.yokogawa.com/Yokogawa_PID_Tuning_Guide_-_csTuner.pdf 